SHORT REPORT

\title{
Prevalence of chronic fatigue syndrome-like caseness in the working population: results from the Maastricht cohort study
}

\author{
M J H Huibers, IJ Kant, G M H Swaen, S V Kasl
}

Occup Environ Med 2004;61:464-466. doi: 10.1136/oem.2003.008912

Aim: To determine the prevalence of chronic fatigue syndrome (CFS)-like caseness in the working population.

Methods: Using data from the prospective Maastricht Cohort Study on Fatigue at Work, the prevalence and incidence of CFS-like cases (employees meeting research criteria for CFS) were determined among 5499 employees who responded to the follow up assessment 3 years and 8 months after baseline.

Results: Of the 5499 employees, 199 (3.6\%) were identified as CFS-like cases. By deleting possible CFS-like cases at baseline, the annual incidence of CFS-like caseness was estimated to be 85 per 10000 . Twenty employees (0.36\%) reported having been diagnosed with CFS by a physician. Conclusions: The prevalence of CFS-like cases (3.6\%) was considerably higher than the prevalence of CFS reported in previous studies $(0.006-3 \%)$. These findings suggest that the CFS-like caseness may be underdetected in the working population and perhaps in other populations as well.

$\mathrm{F}$ tigue is a common complaint in the working population, with a reported prevalence of $22 \%{ }^{1}$ Fatigue can best be understood as a continuum, ranging from mild complaints frequently seen in the community to severe, disabling fatigue like chronic fatigue syndrome (CFS). ${ }^{2}$ When fatigue becomes severe and persistent, it may lead to long term sick leave ${ }^{3}$ and work disability. ${ }^{4}$

Although the body of literature on fatigue in the working population is growing substantially, the relation to CFS has barely received any attention in occupational health. CFS is characterised by persistent medically unexplained fatigue for at least six months, several unexplained symptoms (for example, sore throat, headache, painful joints), and severe functional impairment, with a reported prevalence ranging from $0.006 \%$ to $3.0 \%$ depending on setting and definitions used. ${ }^{5}$ The cause of CFS is unknown and most patients do not recover spontaneously. ${ }^{5}$

The aim of the present report is to determine the prevalence of CFS-like caseness in the working population. We believe we are the first to do so.

\section{METHODS}

\section{Design and study population}

We report data from the prospective Maastricht Cohort Study on Fatigue at Work (MCS). In May 1998, 26978 employees from 45 companies and organisations representing the main work sectors in the Netherlands were invited to participate in the MCS. A total of 12161 employees (45\%) responded by providing informed consent and completing the postal selfreport questionnaire at baseline. There were no demographic differences between respondents and non-respondents at baseline. Further details on the MCS are described elsewhere. $^{6}$

The present report is based on follow up data that were obtained 3 years and 8 months after baseline measurement. Variables necessary to determine whether employees met research criteria for CFS were only available at this point. Consequently, 5499 employees who completed all CFS related questions were included in the analysis. Respondents at follow up $(\mathrm{n}=5499)$ on average had a higher educational level compared to baseline respondents who did not complete follow up $(n=6662)$. Other demographic factors did not differ considerably.

\section{Study variables}

Fatigue was assessed with the subscale fatigue severity of the Checklist Individual Strength (CIS). ${ }^{7}$ Scores can range from 8 to 56, with higher scores indicating a higher severity of fatigue. Functional impairment was assessed with the subscale physical functioning of the Short Form Health Survey (SF-36). ${ }^{8}$ Scores can range from 0 to 100, with higher scores indicating higher levels of physical functioning. Burnout was assessed with the subscale exhaustion of the Maslach Burnout Inventory - General Survey (MBI-GS). ${ }^{9}$ Scores can range from 0 to 6 , with higher scores indicating higher levels of work related emotional exhaustion. Psychological distress was assessed with the Hospital Anxiety and Depression Scale (HAD) that consists of an anxiety and a depression scale. ${ }^{10}$ Scores can range from 0 to 21 on each scale, with higher scores indicating higher levels of anxiety and/or depression. A score of 11 or higher on either of the two scales is indicative of being a psychological distress case. ${ }^{11}$ Other variables included self-reported absence from work and demographic variables.

\section{CFS-like caseness and CFS diagnosis}

Employees were identified as CFS-like cases if they met all of the following research criteria that approximate the CDC criteria for CFS: ${ }^{12}$ a CIS score of 40 or higher, a self-reported duration of fatigue complaints of six months or more, a SF-36 score on physical functioning of 60 or lower, and the selfreported presence of four or more CFS symptoms during the past six months, in line with previous studies of CFS..$^{13}$ Somatic or psychiatric conditions that might explain fatigue complaints could not be excluded. Since CFS criteria could only be fully assessed at follow up, potential CFS-like cases at baseline were identified based on provisional criteria: a CIS score of 40 or higher at baseline, scoring "yes" on at least one of two questions on health related impairment at baseline and a reported duration of fatigue complaints of four years or more at follow up. It is emphasised here that CFS-like cases did not necessarily qualify as CFS patients: a CFS diagnosis can only be made by a physician after a sufficient physical examination. However, CFS-like caseness is regarded as a good proxy for true CFS. ${ }^{15}$ 


\section{Main messages}

- Fatigue is a common complaint in the working population. The prevalence of CFS in the working population is unknown.

- In total, $3.6 \%$ of the 5499 employees screened met the research criteria for CFS. Only $0.36 \%$ of the employees reported having been diagnosed with CFS.

- The prevalence of employees meeting research criteria for CFS is considerably higher than previously reported prevalences of CFS.

In addition, the follow up questionnaire included the following question: "Did you at any time receive a diagnosis of chronic fatigue syndrome or $\mathrm{ME}$ from a qualified physician?". However, this information was not used to determine CFS-like caseness.

\section{Statistical analysis}

Differences between CFS-like employees and non-CFS employees were tested using multiple logistic regression models, adjusting for demographic factors.

\section{RESULTS}

\section{Prevalence and incidence}

Of the 5499 employees at follow up, 199 employees (3.6\%) met the CFS research criteria (see table 1). Of these 199 CFSlike cases, 28 employees might have been CFS-like cases at baseline. By deleting these 28 employees from the population at risk, we estimated the yearly incidence of CFS-like

\section{Policy implications}

- Findings suggest that CFS-like caseness may be underdetected in the working population and perhaps in other populations as well.

caseness to be 85 cases per 10000 . Prevalence and incidence indicated a mean duration of CFS-like caseness of approximately four years $(\mathrm{P}=\mathrm{I} \times \mathrm{D})$.

Of the 20 employees $(0.36 \%)$ who reported a lifetime diagnosis of CFS, only two employees were identified as CFSlike cases.

\section{Characteristics}

There were no gender differences between CFS-like employees and non-CFS employees (see table 1). Compared to employees who did not meet CFS research criteria, CFS-like cases on average were older, had a lower education, and were more likely to suffer from burnout, depression, or anxiety, be a psychological distress case (HAD), or be absent from work at follow up. In the group of employees who reported having received a CFS diagnosis, the mean age was 43.6 (SD 8.1); 11 employees $(55 \%)$ were female; and the majority had a high $(45 \%)$ or medium educational level (45\%; not in table).

\section{DISCUSSION}

We found a prevalence of CFS-like caseness in the working population of $3.6 \%$. Although prevalence estimates vary greatly due to differences in CFS definitions and study settings, the criteria based prevalence we found is still

Table 1 Prevalence, incidence, and characteristics of CFS-like cases in the Maastricht cohort study at follow up

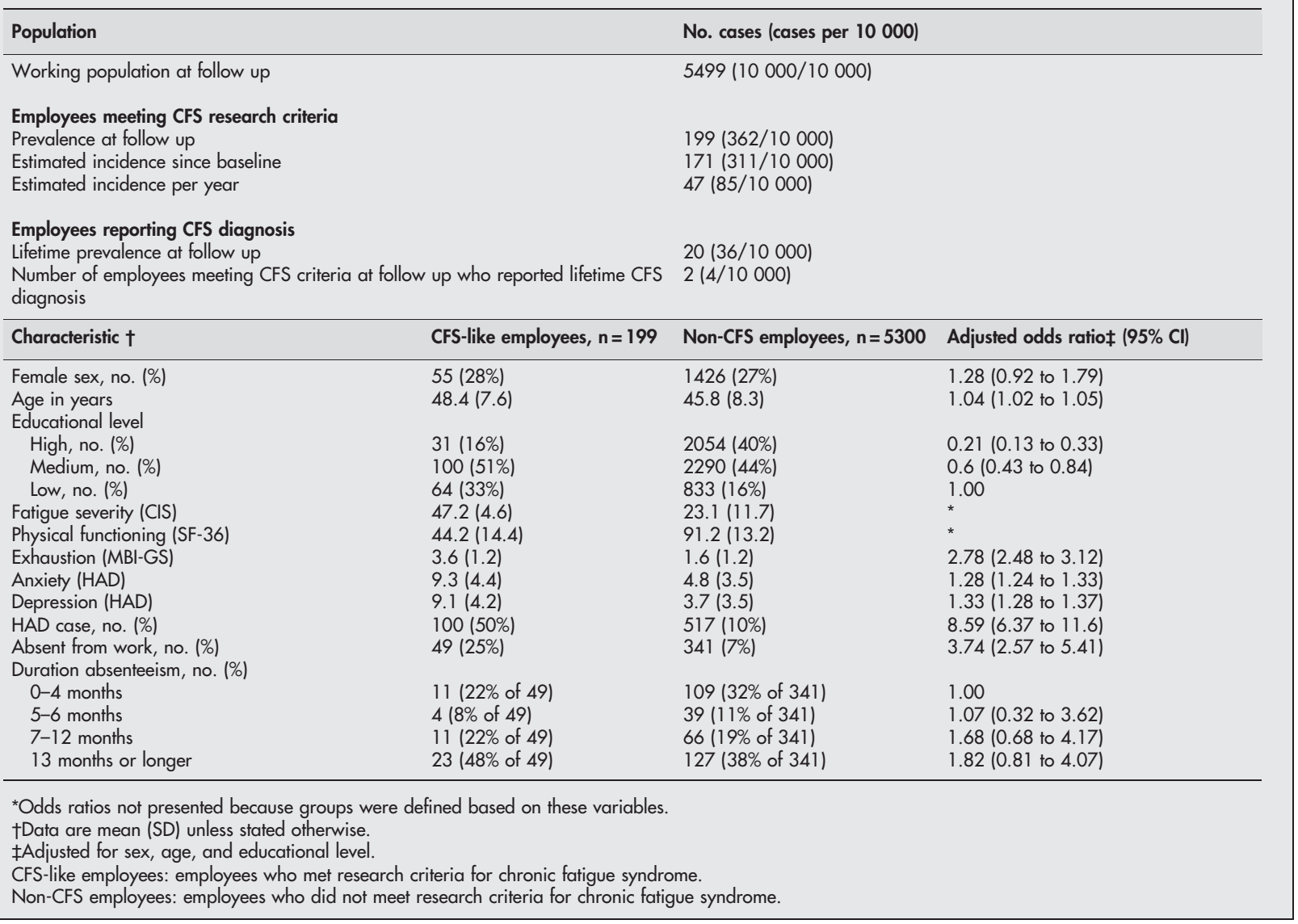


considerably higher than CFS prevalences reported across previous studies. ${ }^{5}$ Also interesting in this context is our finding that only $0.36 \%$ of the employees claimed to have received a CFS diagnosis, while 10 times as many employees (3.6\%) actually met CFS criteria, with a poor overlap between both groups. It was surprising to find that there were no gender differences between CFS-like and non-CFS employees since most diagnosed CFS patients are women. ${ }^{5}$

Most studies of the prevalence of CFS are conducted in clinical settings among diagnosed and/or help-seeking CFS patients, which inevitably introduces self-selection bias. The major advantage of the present report is that we detected (undiagnosed) CFS-like cases using research criteria free from bias regarding healthcare seeking or illness recognition by doctors and patients. This screening procedure may explain the high prevalence of CFS-like caseness we found in contrast to previous studies and the number of employees reporting to have received a CFS diagnosis. The prevalence reported here may even be an underestimation. Prospective studies in the working population are usually biased by the healthy worker effect. In the Maastricht Cohort Study, fatigued employees at baseline were more likely to drop out in time than non-fatigued employees. ${ }^{6}$ On the other hand, the use of self-report questionnaires clearly has limitations in the assessment of CFS. A major disadvantage of the present report is that we could not exclude somatic or psychiatric conditions that might explain fatigue, which in turn may have led to an overestimation of CFS-like caseness. The considerable percentage of psychological distress cases among CFS-like employees seems to confirm this notion, although HAD caseness does not qualify as indicator of a psychiatric disorder that might explain fatigue. Furthermore, we are not sure if the selection of potential CFS-like cases at baseline was accurate since we used a provisional set of necessary but insufficient criteria.

Our findings lead us to suggest that CFS-like caseness may be underdetected in the working population and possibly in other populations as well. A possible explanation would be that the road to receiving a diagnosis of CFS usually takes a lengthy process of vigorous healthcare seeking. Whether the male gender, educational level, and active work status of most undiagnosed CFS-like cases in this study play a role in this is unclear.

\section{ACKNOWLEDGEMENTS}

This study was funded by the Health Research and Development Council (ZorgOnderzoek Nederland), the Netherlands (grant no. 2830180). The Maastricht Cohort Study is part of the Netherlands concerted action on "Fatigue at Work" granted by The Netherlands Organization for Scientific Research (NWO).

\section{Authors' affiliations}

M J H Huibers, Department of Medical, Clinical \& Experimental Psychology, Maastricht University, Netherlands

IJ Kant, G M H Swaen, Department of Epidemiology, Maastricht University, Netherlands

S V Kasl, Department of Epidemiology and Public Health, Yale University School of Medicine, New Haven, CT, USA

Correspondence to: Dr M J H Huibers, Department of Medical, Clinical \& Experimental Psychology, Maastricht University, PO Box 616, 6200 MD Maastricht, Netherlands; marcus.huibers@hag.unimaas.nl

Accepted 5 September 2003

\section{REFERENCES}

1 Bültmann U, Kant IJ, Kasl SV, et al. Fatigue and psychological distress in the working population: psychometrics, prevalence and correlates. J Psychosom Res 2002;52:445-52.

2 Lewis G, Wessely S. The epidemiology of fatigue: more questions than answers. J Epidemiol Community Health 1992;46:92-7.

3 Janssen N, Kant IJ, Swaen GMH, et al. Fatigue as a predictor of sickness absence: results from the Maastricht cohort study on fatigue at work. Occup Environ Med 2003;60(suppl 1):i71-6.

4 Amelsvoort van LGPM, Kant IJ, Beurskens AJHM, et al. Fatigue as a predictor of work disability. Occup Environ Med 2002;59:712-13.

5 Afari N, Buchwald D. Chronic fatigue syndrome: a review. Am J Psychiatry 2003; 160:221-36

6 Kant IJ, Bültmann U, Schroer CAP, et al. An epidemiological approach to study fatigue in the working population: The Maastricht Cohort Study. Occup Environ Med 2003:60(suppl 1):i32-9.

7 Beurskens AJHM, Bültmann U, Kant IJ, et al. Fatigue among working people: validity of a questionnaire measure. Occup Environ Med 2000;57:353-7.

8 Ware JE Jr, Sherbourne CD. The MOS 36-item short-form health survey (SF36). I. Conceptual framework and item selection. Med Care 1992;30:473-83.

9 Schaufeli WB, Leiter MP, Maslach C, et al. The Maslach Burnout InventoryGeneral Survey. In: Maslach C, Jackson SE, eds. Maslach Burnout Inventory Manual. Palo Alto, CA: Consulting Psychologists Press, 1996.

10 Zigmond AS, Snaith RP. The Hospital Anxiety and Depression Scale. Acta Psychiatr Scand 1983;67:361-70.

11 Wilkinson MJB, Barczak P. Psychiatric screening in general practice: comparison of the general health questionnaire and the hospital anxiety and depression scale. JR Coll Gen Pract 1988;38:311-13.

12 Fukuda K, Straus SE, Hickie I, et al. The chronic fatigue syndrome: a comprehensive approach to its definition and study. International Chronic Fatigue Syndrome Study Group. Ann Intern Med 1994;121:953-9.

13 Powell P, Bentall RP, Nye FJ, et al. Randomised controlled trial of patient education to encourage graded exercise in chronic fatigue syndrome. BMJ 2001;322:387-90.

14 Prins JB, Bleijenberg G, Bazelmans E, et al. Cognitive behaviour therapy for chronic fatigue syndrome: a multicentre randomised controlled trial. Lancet 2001;357:841-7.

15 Jason LA, Taylor RR. Applying cluster analysis to define a typology of chronic fatigue syndrome in a medically-evaluated, random community sample. Psychol Health 2002;17:323-37. 